Original Research Paper

\title{
Space Distribution of Highland Barley GNS and its Relationship with Environmental Factors in the Qinghai-Tibet Plateau
}

\author{
Xibo Feng, Gaihua Wang and Jianlin Wang \\ Xizang Agriculture and Animal Husbandry College, No. 100 YuCai West Road, 860000, \\ Linzhi City, Tibet Autonomous Region, China
}

\author{
Article history \\ Received: 16-03-2018 \\ Revised: 16-04-2018 \\ Accepted: 25-04-2018 \\ Corresponding Author: \\ Jianlin Wang \\ Xizang Agriculture and Animal \\ Husbandry College, No. 100 \\ YuCai West Road, 860000, \\ Linzhi City, Tibet Autonomous \\ Region, China \\ Email: 540899682@qq.com
}

\begin{abstract}
In order to study the space distribution of Grain Number per spike (GNS) and its relationship with the environmental factors in the Qinghai-Tibet Plateau, 83 data samples from different ecological areas were selected for GNS inverstigation. Meanwhile, the seeds of highland barley were harvested and planted in the farm of Xizang Agriculture and Animal Husbandry College; the varieties without genetic differences were selected and their GNS was analyzed with the local geography and climate factors. The results shows that: (1) In the horizontal direction, GNS forms the high value area of Central and South Tibetan taking Qiongjie and Longzi as the center; (2) In the vertical direction, GNS appears with double high value areas at the altitude of $3900 \mathrm{~m}-4200 \mathrm{~m}$ and above $4500 \mathrm{~m}$ respectively and the corresponding GNS is $52.9 \pm 17.6$ and $57.2 \pm 0.2$ respectively; (3) the environmental factors influencing GNS are in the order that soil total nitrogen $>$ soil available nitrogen $>$ the average precipitation at heading-filling stage $>$ altitude $>$ soil organic matter $>$ soil total phosphorus $>$ the average rainfall at jointing-heading stage $>$ soil available potassium $>$ the average rainfall at tillering-jointing stage.
\end{abstract}

Keywords: Qinghai-Tibet Plateau, Highland Barley, Grain Number Per Spike, Distribution Pattern, Environmental Factor

\section{Introduction}

The Qinghai-Tibet Plateau is the largest plateau in China and the highest plateau of the world with complicated ecological environment. It is called "the roof of the world" and "earth's third pole" (Cheng et al., 2017) and is one of the origin centers of pioneering crop barley (Hordeum vulgare L.) (Dai et al., 2014; 2012). Its main planting crop is highland barley, also known as naked barley and hulless barley (Hordeum vulgare Linn. var. nudum Hook.f.) and the Tibetan language is called "Nai". Highland Barley is one of the important foods of Tibetans in Xizang, Qinghai, Gansu and Sichuan with a long planting history and large planting area, up to more than $80 \%$ of the crop planting area (Ma, 2000). Grain Number per Spike (GNS) is an important component factor of highland barley yield, its affecting factors are more and with complicated mechanism (Gesangzhuoma, 1995). Li et al. (2006) thought barley GNS was associated with genetic factor; Du et al. (2002) thought the temperature of highland barley at booting-heading stage and the precipitation at flowering-milk stage in
Xigaze of Xizang had a significant effect on the GNS; Chalak et al. (2015) thought the rainfall before and after the flowering had a great influence on barley GNS; Prystupa et al. (2004) thought early Nitrogen(N) influenced GNS; Abbate et al. (1995) used the ratio of PAR obtained before and after the flowering and the average temperature to describe GNS linearly. Abeledo et al. (2008; García et al., 2003) thought GNS was the result of environment and species interactions. Zou et al. (2009) combined the genetic factors and environmental factors (light, temperature, water and fertilizer) organically and established the mathematic model of the barley yield to make corresponding prediction for GNS.

The above study is given priority to skin barley with small area coverage, less sample quantity; the geographical factors, for example, the effects of altitude, latitude and longitude on the GNS were not researched. The QinghaiTibet Plateau crosses more than 10 latitudes and 30 longitudes and the altitude is from $200 \mathrm{~m}$ to over $6000 \mathrm{~m}$, which provides a natural model for the large-scale and 
three-dimensional highland barley GNS research (Wang et al., 2014). Taking the sample data in the large-scale scope of Qinghai-Tibet Plateau as support, the paper adopts the research methods with the combination of agricultural ecology and geography through the establishment of stepwise regression and advantages (dominant) model to reveal the space distribution characteristics of highland barley GNS in the horizontal and vertical directions and the influence of different environmental factors on it, to guide the highland barley breeding and cultivation.

\section{Materials and Methods}

\section{Study Area}

The study area stretches the mountain steppe, alpine meadow steppe, alpine scrub meadows, mountain coniferous forest, mountain scrub grassland, mountain desert and semi-desert, involved in Sichuan, Gansu, Qinghai and Tibet. The sunshine hours in growth period are $1642-3393 \mathrm{~h}$, the temperatures in growth period are $-0.6 \sim 12.9^{\circ} \mathrm{C}$, the rainfall in growth period is $150-890 \mathrm{~mm}$.

\section{Highland Barley Collection and Sample Points Arrangement}

In July-September of 2014 and July-September of 2015, 83 sample points were set in the different planting areas (northern latitude $27-38^{\circ} \mathrm{C}$, east longitude $79-104^{\circ} \mathrm{C}$ ) of Qinghai-Tibet Plateau and a total of 83 varieties (promotion) were collected from the local peasant. Among them, there are 67 peasant varieties and 16 promotion varieties. The specific geographic location was shown in Fig. 1. When the samples were collected, 20 strains of highland barley were randomly pulled out for GNS investigation.

\section{Soil Samples Collection and Analysis}

When highland barley was collected for GNS investigation at each sample point, 5-6 sample quadrats was set; 3 soil samples in the plough layer $(0-30 \mathrm{~cm})$ were collected randomly from each sample quadrat (the area was not less than $20 \mathrm{~m}^{2}$ ) and put them into the soil bag. After drying in the lab, the soil was grinded fine for $1 \mathrm{~mm}$ sieving to determine total $\mathrm{n}$, total phosphorus, total potassium, available n, available phosphorus, available potassium, $\mathrm{pH}$ and soil. Among them, determination of the total nitrogen of soil and available nitrogen adopted semi-micro kjeldahl method (Feng et al., 2014); determination of total phosphorus and available phosphorus of soil adopted Vanadium molybdate blue colorimetric method (Lu, 1991); determination of total potassium and available potassium adopted flame photometry; determination of organic matter used potassium dichromate oxidation-heating method (Shuang and Nisana, 2016) and $\mathrm{pH}$ value was determined by potentiometry.

\section{Test Design}

83 varieties with different germplasm were planted in the farm of Xizang Agriculture and Animal Husbandry College (March-August, 2015 and March-August, 2016). The area was $6 \mathrm{~m} \times 4 \mathrm{~m}=24 \mathrm{~m}^{2}$ with random block arrangement with 3 times repeat. The management was the same with local field. After harvest; each community chose 10 strains for GNS statistics.

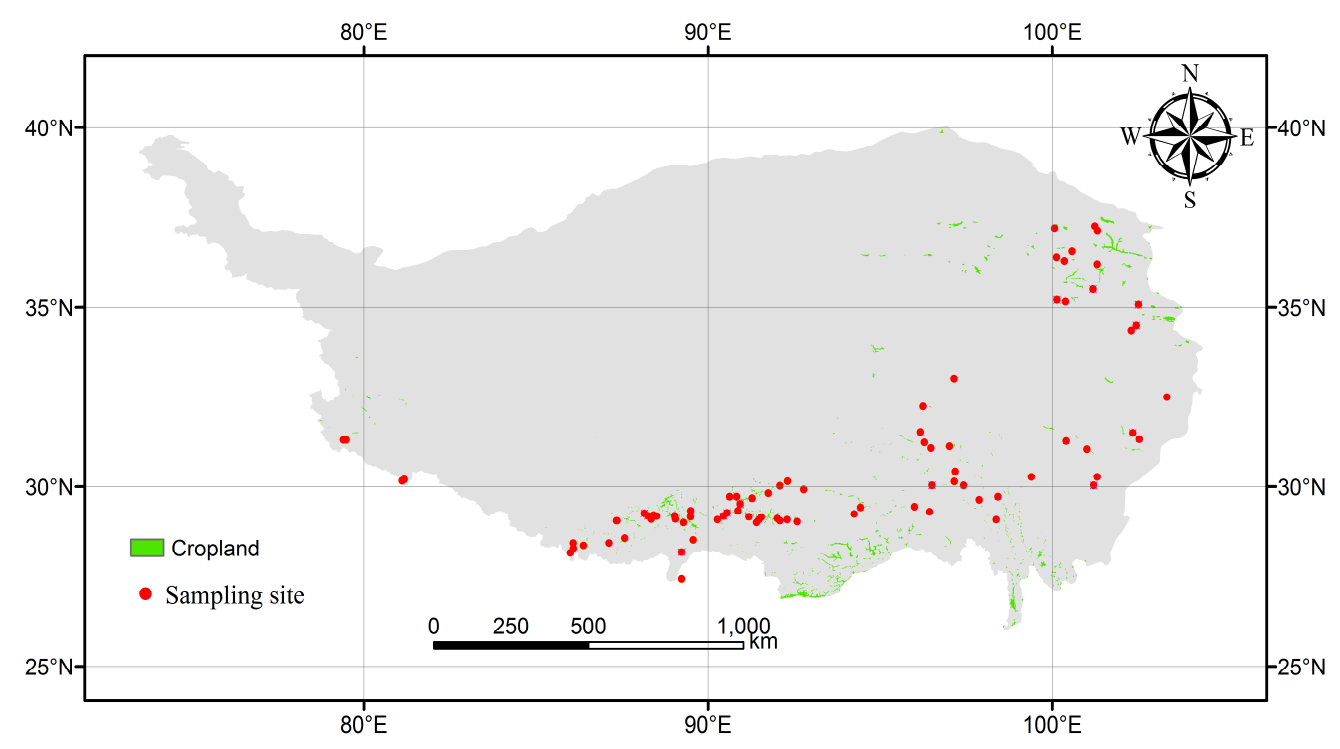

Fig. 1: Distributions of sampling points 




Fig. 2: Distribution of highland barley GNS in the Qinghai-Tibet plateau

\section{Data Analysis and Processing}

(1) Using ARCGIS9.3 software made space distribution (shown in Fig. 2) of highland barley GNS for the 83 sample points. (2) In order to study the effect of environmental factors on highland barley GNS, the interference of variety factor (genetic factor) should be eliminated. In view of the dozens of latitude and longitude and thousands of meters of altitude gradient of Qinghai-Tibet Plateau, we chose dozens of sample points and tens of varieties for planting experiment. This study was mainly that different highland barley varieties in different sample points were planted in the same environment conditions. Through the variance analysis and multiple comparisons, the samples (eliminate the interference of variety factor) without significant GNS difference are chosen and using the GNS data of origin and geography, soil and climate data of origin (note: Climate data comes from China's national meteorological information center) to establish data model and compare the contribution of each environmental factor to GNS.

\section{Results}

Distribution Characteristics of Highland Barley GNS in the Qinghai-Tibet Plateau

\section{The Horizontal Distribution Characteristics of GNS}

As shown in Fig. 2, the high value area of highland barley in the Qinghai-Tibet Plateau is mainly in longitude $91.0-92.5^{\circ}$ and north latitude $29.0-31.0^{\circ}$ and the high value area of central and southern Tibet taking
Qiongjie, Longzi, Jiacha and Shangri county of Xizang, where the average GNS is around 71.9 \pm 4.0 .

\section{The Vertical Distribution Characteristics of GNS}

Vertically, GNS presented "bimodal curve" type distribution pattern with the increasing of altitude (Table 1). That is, under the altitude of 3000-3300 m, GNS changes smoothly with the change of altitude. Above the altitude of 2700-3000 m, highland barley GNS increases with the increasing of altitude; it reaches a higher value area at altitude of $3900-4200 \mathrm{~m}$ and the average GNS is $52.9 \pm 17.6$. And then, GNS decreases with the increasing of altitude and reaches the least value at the altitude of 4200-4500 m. After that, GNS increases gradually with the increase of altitude and reaches the peak value at above $4500 \mathrm{~m}$ with $57.2 \pm 0.2$. In the area that the altitude is less than $2700 \mathrm{~m}$ and more than $4200 \mathrm{~m}$, the variation coefficient of highland barley GNS is small; in the area that the altitude is $2700-4200 \mathrm{~m}$, the variation coefficient of highland barley GNS is large (Table 1)

\section{The Relationship of Highland Barley GNS in the Qinghai-Tibet Plateau and Environmental Factors}

After the planting of 83 sample points on the farm of Xizang Agriculture and Animal Husbandry College, their GNS was for variance analysis. The results showed that the amplitude of highland barley GNS was 22.2-86.9, the average was $49.7 \pm 17.0$ and the variation coefficient was $34.2 \%$, different sources of highland barley varieties GNS were extremely significant different (Table 2). After multiple comparisons to the 83 varieties from the 83 sample points, it can be found that 47 varieties had no significant difference. 
Table 1: The distribution of highland barley GNS in different elevation gradient

\begin{tabular}{lccc}
\hline Altitude $(\mathrm{m})$ & Number of sites & Average (\%) & CV (\%) \\
\hline$<2700$ & 2 & $45.3 \pm 6.9$ & 15.29 \\
$2700 \sim 3000$ & 6 & $44.2 \pm 14.9$ & 33.74 \\
$3000 \sim 3300$ & 17 & $47.8 \pm 14.4$ & 30.03 \\
$3300 \sim 3600$ & 9 & $49.5 \pm 19.0$ & 38.32 \\
$3600 \sim 3900$ & 27 & $49.4 \pm 19.0$ & 38.39 \\
$3900 \sim 4200$ & 14 & $52.9 \pm 17.6$ & 33.20 \\
$4200 \sim 4500$ & 6 & $48.7 \pm 4.8$ & 9.92 \\
$>4500$ & 2 & $57.2 \pm 0.2$ & 0.30 \\
Total & 83 & $49.4 \pm 16.1$ & 32.71 \\
\hline
\end{tabular}

Table 2: F-test of highland barley variety GNS from different sources

\begin{tabular}{lcrlll}
\hline Variation & SS & DF & MS & F & p \\
\hline Inter-blocks & 524.4046 & 2 & 262.2023 & 2.597 & 0.0776 \\
nter-treatments & 54397.4563 & 82 & 663.3836 & 6.569 & 0.0023 \\
Error & 16561.1688 & 164 & 100.9827 & & \\
Total CV & 71483.0296 & 248 & & & \\
\hline
\end{tabular}

\section{The Relationship of Highland Barley GNS and Geographical Factors}

Taking geographic longitude $\left(X_{1}\right)$, latitude $\left(X_{2}\right)$ and altitude $\left(X_{3}\right)$ as the independent variables, highland barley $\operatorname{GNS}(Y)$ as the dependent variable to establish the regression equation as follows:

$$
\begin{aligned}
& Y=66.4289+0.400 X_{1}-0.3416 X_{2}-0.5615 X_{3} \\
& \left(\begin{array}{l}
N=47 R=0.3399 R_{1}=0.1399 \\
R_{2}=-0.4614 R_{3}=-0.5416^{*}
\end{array}\right)
\end{aligned}
$$

It can be seen from Equation (1) that highland barley GNS $(Y)$ has positive correlation with geographic longitude $\left(X_{1}\right)$, has negative correlation with geographic latitude $\left(X_{2}\right)$ and altitude $\left(X_{3}\right)$; among them, the effect of altitude on highland barley GNS is prominent. For the reason that the regression equation does not make $F$ test, it shows that the combination of each geographical factor has no significant effect on highland barley GNS.

\section{The Relationship of Highland Barley GNS and Climatic Factors}

Taking highland barley $\operatorname{GNS}(Y)$ as the dependent variable, sunshine hours in growth $\operatorname{period}\left(X_{1}\right)$, evaporation in growth period $\left(X_{2}\right)$, accumulated temperature $\geq 0^{\circ} \mathrm{C}$ in growth period $\left(X_{3}\right)$, accumulated temperature $\geq 10^{\circ} \mathrm{C}$ in growth period $\left(X_{4}\right)$, temperature in growth period $\left(X_{5}\right)$, the relative humidity in growth period $\left(X_{6}\right)$, rainfall in growth period $\left(X_{7}\right)$, the average temperature at emergence-tillering stage $\left(X_{8}\right)$, the average temperature at tillering-joining stage $\left(X_{9)}\right.$, the average temperature joining-heading stage $\left(X_{10}\right)$, the average temperature at heading-filling stage $\left(X_{11}\right)$, the average temperature fiiling-mature period $\left(X_{12}\right)$, daily range of the average temperature at emergencetillering stage $\left(X_{13}\right)$, daily range of the average temperature at tillering-jointing stage $\left(X_{14}\right)$, daily range of the average temperature in jointing-heading period $\left(X_{15}\right)$, daily range of the average temperature at heading-filling stage $\left(X_{16}\right)$, daily range of the average temperature in filling-mature period $\left(X_{17}\right)$, the average relative humidity at emergence-tillering stage $\left(X_{18}\right)$, the average relative humidity at tillering-jointing stage $\left(X_{19}\right)$, the average relative humidity in jointingheading period $\left(X_{20}\right)$, the average relative humidity at heading-filling stage $\left(X_{21}\right)$, the average relative humidity in filling-mature period $\left(X_{22}\right)$, the average precipitation at emergence-tillering stage $\left(X_{23}\right)$, the average precipitation at tillering-jointing stage $\left(X_{24}\right)$, the average precipitation at jointing-tillering stage $\left(X_{25}\right)$, the average precipitation in tillering-filling period $\left(X_{26}\right)$ and the average precipitation in filling-mature period $\left(X_{27}\right)$ as the independent variables, the equation was established as follows through stepwise regression:

$$
\begin{aligned}
& Y=65.7023-0.0056 X_{4}+0.2892 X_{6}+0.4644 X_{12} \\
& +0.3139 X_{19}-0.9923 X_{22}+0.2530 X_{24}-0.3657 X_{25} \\
& +0.5302 X_{26}-0.1890 X_{27} \\
& \left(\begin{array}{l}
N=47 R=0.6737 R_{4}=-0.2078 R_{6}=0.3549 \\
R_{12}=0.1517 R_{19}=0.2752 R_{22}=-0.4206 R_{24}=-0.4392^{*} \\
R_{25}=-0.5898^{* *} R_{26}=0.4916^{*} R_{27}=-0.2219
\end{array}\right)
\end{aligned}
$$


It can be found from Equation (2) that the main climate factors effecting highland barley are $X_{4}, X_{6}, X_{12}, X_{19}, X_{22}$, $X_{24}, X_{25}, X_{26}$ and $X_{27}$. The effects of sunshine hours in growth period $\left(X_{1}\right)$, evaporation in growth period $\left(X_{2}\right)$ and accumulated temperature $\geq 0{ }^{\circ} \mathrm{C}$ in growth period $\left(X_{3}\right)$ are small. Meanwhile, it can also be found from Equation (2) that highland barley GNS and the partial correlation coefficient of $X_{26}$ reach the difference level of positive correlation; highland barley GNS and $X_{24}$ and $X_{25}$ reach the difference level of significant and extremely significant negative correlation; highland barley GNS and the partial correlation coefficient of $X_{4}$, $X_{6}, X_{12}, X_{19}, X_{22}$ and $X_{27}$ do not reach the difference level of significance $(p<0.05) . F$ test of the regression equation was not significant, which indicates that the combination of climate factors on the impact of barley GNS not reached significant level $(p<0.05)$.

\section{The Relationship of Highland Barley GNS and Soil Factors}

Taking highland barley GNS $(Y)$ as the dependent variable, soil organic matter $\left(X_{1}\right)$, total $N\left(X_{2}\right)$, total phosphorus $\left(X_{3}\right)$, total potassium $\left(X_{4}\right)$, soil available $N$ $\left(X_{5}\right)$, available phosphorus $\left(X_{6}\right)$, available potassium $\left(X_{7}\right)$ and soil $\mathrm{PH}$ as the independent variable, the equation is as follows through stepwise regression:

$$
\begin{gathered}
Y=37.0299-2.4259 X_{1}+49.4526 X_{2} \\
\quad+167.4792 X_{3}-0.0222 X_{5}-0.0386 X_{7} \\
\left(\begin{array}{l}
N=47 R=0.9060^{* *} R_{1}=-0.5688^{* *} R_{2}=0.9032^{* *} \\
R_{3}=0.6034^{* *} R_{5}=-0.6895^{* *} R_{7}=-0.4535^{* *}
\end{array}\right)
\end{gathered}
$$

It can be found from Equation (3) that the main soil factors influencing highland barley $\operatorname{GNS}(Y)$ are $X_{1}$,
$X_{2}$ 、 total phosphorus $X_{3}, X_{5}$ and $X_{7}$; while, the effect of $X_{4}, X_{6}$ and $X_{8}$ is small. It can also be seen from Equation (3) that highland barley GNS has extremely significant positive correlation with $X_{2}$ and $X_{3}$ and has extremely significant negative correlation with $X_{1}, X_{5}$ and $X_{7}$. The regression equation passes $F$ test, which indicates that highland barley GNS increases with the increasing of $X_{2}$ and $X_{3}$ and the decreasing of $X_{1}, X_{5}$ and $X_{7}$.

\section{The Relationship of Highland Barley GNS and Comprehensive Factors}

According to the above analysis, highland barley GNS and the partial correlation coefficient of the average precipitation at heading-filling stage $\left(X_{26}\right)$, total $N\left(X_{2}\right)$ and total phosphorus $\left(X_{3}\right)$ reaches the difference level of significant or extremely significant positive correlation. Highland barley GNS and the partial correlation coefficient of the average precipitation at tillering-jointing stage $\left(X_{24}\right)$, the average precipitation at jointing-tillering stage $\left(X_{25}\right)$, soil organic matter $\left(X_{1}\right)$, available $\mathrm{N}\left(X_{5}\right)$, available potassium $\left(X_{7}\right)$ and altitude $\left(X_{8}\right)$ reach the difference level of significant or extremely significant negative correlation. Therefore, we use advantages (leading) analysis to make importance analysis to the 9 variables (soil organic matter $X(1)$, total $\mathrm{N}$ of soil $X(2)$, soil total phosphorus $X(3)$, soil available $\mathrm{N} X(4)$, soil available potassium $X(5)$, the average rainfall at tillering-jointing stage $X(6)$, the average rainfall at jointing- heading stage $X(7)$, the average rainfall at heading - filling stage $X(8)$, altitude $X(9)$ affecting highland barley GNS significantly, the order is total $\mathrm{N}$ of soil $>$ soil available $\mathrm{N}>$ the average rainfall at heading - filling stage $>$ altitude $>$ soil organic matter $>$ soil total phosphorus $>$ the average rainfall at jointingheading stage $>$ soil available potassium $>$ the average

\begin{tabular}{|c|c|c|c|c|c|c|c|c|c|}
\hline Models & $X(1)$ & $X(2)$ & $X(3)$ & $X(4)$ & $X(5)$ & $X(6)$ & $X(7)$ & $X(8)$ & $X(9)$ \\
\hline Single factor & 0.0278 & 0.3619 & 0.0338 & 0.0465 & 0.0075 & 0.0028 & 0.0253 & 0.0998 & 0.0728 \\
\hline One order interaction & 0.0740 & 0.4541 & 0.0795 & 0.1012 & 0.0494 & 0.0185 & 0.0389 & 0.0968 & 0.0711 \\
\hline Two orders interaction & 0.1094 & 0.4593 & 0.1130 & 0.1371 & 0.0838 & 0.0599 & 0.0748 & 0.1019 & 0.0864 \\
\hline Three orders interaction & 0.1369 & 0.4321 & 0.1373 & 0.1613 & 0.1123 & 0.1065 & 0.1170 & 0.1221 & 0.1013 \\
\hline Four orders interaction & 0.1624 & 0.3840 & 0.1577 & 0.1796 & 0.1385 & 0.1433 & 0.1502 & 0.1455 & 0.1232 \\
\hline Five orders interaction & 0.1840 & 0.3242 & 0.1755 & 0.1932 & 0.1624 & 0.1675 & 0.1713 & 0.1656 & 0.1457 \\
\hline Six orders interaction & 0.1938 & 0.2600 & 0.1889 & 0.1985 & 0.1807 & 0.1814 & 0.1825 & 0.1795 & 0.1587 \\
\hline Seven orders interaction & 0.1915 & 0.1979 & 0.1910 & 0.1919 & 0.1901 & 0.1901 & 0.1902 & 0.1899 & 0.1786 \\
\hline Eight orders interaction & 0.1350 & 0.3592 & 0.1346 & 0.1512 & 0.1156 & 0.1088 & 0.1188 & 0.1376 & 0.1213 \\
\hline Nine orders interaction & 0.0278 & 0.3619 & 0.0338 & 0.0465 & 0.0075 & 0.0028 & 0.0253 & 0.0998 & 0.0776 \\
\hline Ten orders interaction & 0.0740 & 0.4541 & 0.0795 & 0.1012 & 0.0494 & 0.0185 & 0.0389 & 0.0968 & 0.0745 \\
\hline Total & 0.1094 & 0.4593 & 0.1130 & 0.1371 & 0.0838 & 0.0599 & 0.0748 & 0.1019 & 0.1011 \\
\hline Percentage (\%) & 10.7100 & 28.4900 & 10.6800 & 11.9900 & 9.1700 & 8.6300 & 9.4200 & 10.9200 & 10.8200 \\
\hline
\end{tabular}
rainfall at tillering-jointing stage (Table 3 ).

Table 3: The contribution of decision factor in each order regression model 


\section{Discussion}

This study results show that soil factors interact with climate factors, in particular, the effect of soil factors on the Qinghai-Tibet Plateau has a great influence on highland barley GNS. Among them, the geographical factor effecting highland barley GNS is altitude gradient; soil factor is the content of total $\mathrm{n}$ and available $\mathrm{n}$; the climate factor is the average precipitation at headingfilling stage. Influenced by these environment factors, highland barley GNS appears a patch shape distribution pattern in the geographical horizontal directions, forming high value areas of highland barley GNS taking Tibet Qiongjie, Longzi, Jiazha and Sangri County as the center; In the vertical direction, the change of highland barley appears "bimodal curve" distribution pattern and forms two high value areas of highland barley above the altitude of 3900-4200 and $4500 \mathrm{~m}$. Causes of this phenomenon may be related to the unique plateau climate factors and the effect of soil difference in the geographical area of Qinghai-Tibet Plateau. That is consistent with the research results that most of scholars thought although barley GNS is mainly controlled by variety characteristics, i.e., control of genetic factors and environmental factors also have obvious influence on it (Abeledo et al., 2008; García et al., 2003; Zou et al., 2009).

Partial correlation analysis shows that highland barley GNS has positive correlation with the average precipitation at heading-filling stage and soil total $\mathrm{n}$ content; and highland barley GNS has negative correlation with the average precipitation at tilleringheading stage, altitude, the content of soil organic matter and available potassium and has no correlation with sunshine hours in the growth period. This study is basically identical with the results of Du et al. (2002; Chalak et al., 2015; Ugarte et al., 2007). However, Guillermoa et al. (2015) thought night temperature increased in the reproductive growth period of barley, GNS decreased; Reinhardt et al. (2013) thought the night temperature was about $30^{\circ} \mathrm{C}$ in the reproductive growth period of barley, GNS decreased significantly. The effect of climate factors on barley GNS is relatively complex, interaction of multiple factors makes a single factor analysis more difficult. In actual experiment, it is difficult to let other variables constant to analyze the effect of a single variable; this is also the limitations of partial correlation analysis. Night temperature increase influences the accumulation of dry matter; extreme high temperature influences pollination and spikelet differentiation and then affects GNS. The research results of Guillermoa et al. (2015; Reinhardt et al., 2013) have no contradiction; on the contrary, large temperature difference in the Qinghai-Tibet Plateau provides corresponding basis for the increase of highland barley GNS. Högy et al. (2013) thought that barley plant in
Stuttgart, Germany, temperature had no significantly effect on barley GNS, which is inconsistent with most research results. Temperature difference is small in Stuttgart, the average temperature all the year round is $10.7^{\circ} \mathrm{C}$ with less rainfall. Temperature is not the restrictive factor for barley growth, the conclusions of Högy et al. (2013) may be related to the special climate factors of Stuttgart.

Many scholars at home and abroad agree that $\mathrm{N}$ rate has positive correlation with barley GNS (Papastylianou, 1995b; Cantero-Martínez et al., 2003; Papastylianou,1995a; Le et al., 1999; Prado et al., 2013; Baethgen et al., 1995) and the results are basically identical with them. Miralles and Richards (2000) though that with the increase of sunshine time, barley GNS in Arapiles increases with it. The study shows that barley GNS is not associated with the sunshine time in growth period. This may be due to Qinghai-Tibet Plateau is located in the low latitudes with longer sunshine time and sunshine time is not the restrictive factor of barley growth and development.

The GNS of barley is one of the most important contributing factors of the yield of barley and it is one of the important indicators of the genetic diversity of barley. According to the spatial distribution pattern of GNS in the Qinghai-Tibet Plateau, they are mostly distributed in the high-altitude areas of the high mountains and valleys in the south of Tibet and eastern Tibet, which is basically consistent with the origin center of barley reported by Ma (2000). Therefore, the spatial distribution regularity of GNS can provide help for the study of the origin and evolution of barley. The results of studies on the impact of environmental factors on the GNS of highland barley indicate that the amount of precipitation at heading stage and nitrogen fertilizer has an important effect on the increase of GNS of the highland barley. Therefore, it is necessary to increase nitrogen fertilizer and timely and reasonable irrigation in order to increase the yield of barley in Qinghai-Tibet Plateau. However, the soil types of the main cultivation areas in the Qinghai-Tibet Plateau are mainly shrub grassland and sub-alpine grassland soils (Hu, 1995). The main characteristics of this soil type are high sand content, poor water retention and fertility conservation. Therefore, reasonable fertilization and irrigation, increase of nitrogen fertilizer and water use efficiency are important issues that need to be resolved in the future.

\section{Conclusion}

Highland barley GNS in the Qinghai-Tibet Plateau appears a patch shape distribution pattern, mainly distributes in the north of Himalayan, central south and east of Tibet in the Transaction Mountain over $3900 \mathrm{~m}$ altitudes. It is located in the high mountain canyon of middle of the Yarlung Zangbo River, the Jinsha River, Nujiang River and Lantsang, is one of the distribution 
centers of barley diversity with complicated and diverse geographical environments. Among them, soil n, altitude gradient, organic matter content and precipitation have great influence on highland barley GNS.

\section{Acknowledgement}

The work is supported by the Natural Science Foundation of China (No. 31360300, 31370458 and 31560362), Key projects of the Tibet Autonomous Region (No. XZXTCX-2015-03 and XBTSZWXK2016-01) and Talent Development Support Plan of Snow Area Talent Project Engineering of Xizang Agriculture and Animal Husbandry College (No. ZLYRCGC-2015-4)

\section{Author's Contributions}

Xi-Bo Feng: Conceived and designed the work that led to the submission, analyzed the data and wrote the paper.

Gai-Hua Wang: Performed the numerical experiments and revised the manuscript.

Jian-Lin Wang: Revised and approved the final manuscript.

\section{Ethics}

The authors declare their responsibility for any ethical issues that may arise after the publication of this manuscript.

\section{Conflict of Interest}

The authors declare that they have no competing interests. The corresponding author affirms that all of the authors have read and approved the manuscript.

\section{References}

Abbate, P.E., F.H. Andrade and J.P. Culot, 1995. The effects of radiation and $n$ on number of grains in wheat. J. Agric. Sci., 124: 351-360.

Abeledo, L.G., D.F. Calderini and G.A. Slafer, 2008. Nitrogen economy in old and modern malting barleys. Field Crops Res., 106: 171-178. DOI: $10.1016 /$ j.fcr.2007.11.006

Baethgen, W.E., C.B. Christianson and A.G. Lamothe, 1995. Nitrogen fertilizer effects on growth, grain yield and yield components of malting barley. Field Crops Res., 43: 87-99.

DOI: 10.1016/0378-4290(95)00034-N

Cantero-Martínez, C., P. Angas and J. Lampurlanés, 2003. Growth, yield and water productivity of barley (Hordeum vulgare L.) affected by tillage and $\mathrm{N}$ fertilization in Mediterranean semiarid, rainfed conditions of Spain. Field Crops Res., 84: 341-357. DOI: $10.1016 / \mathrm{S} 0378-4290(03) 00101-1$
Chalak, L., R. Mzid, W. Rizk, H. Hmedeh and R. Kabalan et al., 2015. Performance of 50 Lebanese Barley landraces (Hordeum vulgare L. subsp. vulgare) in two locations under rainfed conditions. Annals Agric. Sci., 60: 325-334. DOI: 10.1016/j.aoas.2015.11.005

Cheng, W.M., S.M. Zhao and Q.H. Ye, 2017. Alpine Geomorphology and Variations on the Tibetan Plateau. 1st Edn., Science Press, ISBN-13: 9787030523167.

Dai, F., E. Nevo, D.Z. Wu, J. Comadran and M. Zhou et al., 2012. Tibet is one of the centers of domestication of cultivated barley. Proc. Nat. Acad. Sci., 109: 16969-16973. DOI: 10.1073/pnas.1215265109

Dai, F., Z.H. Chen, X.L.Wang, Z. Li and G. Jin et al., 2014. Transcriptome profiling reveals mosaic genomic origins of modern cultivated barley. Proc. Nat. Acad. Sci., 111: 13403-13408. DOI: $10.1073 /$ pnas. 1414335111

Du, J., Gesangzhuoma and Suolangouzhu, 2002. Effects of meteorological conditions on grain number of spring wheat in Plateau. Tibet Sci. Technol., 4: 47-49.

Feng, J.W., Y. Yang and S.H. Zhu, 2014. Study on the improvement of soil total nitrogen using semi-micro Kjeldahl method. Sci. Technol. Res., 23: 391-393.

García, F., M.B. García, J.L. Molina-Cano and G.A. Slafer, 2003. Yield stability and development in two- and six-rowed winter barleys under Mediterranean conditions. Field Crops Res., 81: 109-119. DOI: 10.1016/S0378-4290(02)00215-0

Gesangzhuoma, 1995. Effects of meteorological factors on spring Highland barley. J. Tibet Agric. Sci. Technol., 17: 1-4.

Guillermoa, G., M.F. Dreccer, D.J. Miralles and R.A. Serrago, 2015. High night temperatures during grain number determination reduce wheat and barley grain yield: A field study. Global Change Biol., 21: 4153-4164. DOI: 10.1111/gcb.13009

Högy, P., C. Poll, S. Marhan, E. Kandeler and A. Fangmeier et al., 2013. Impacts of temperature increase and change in precipitation pattern on crop yield and yield quality of barley. Food Chem., 136: 1470-1477. DOI: 10.1016/j.foodchem.2012.09.056

$\mathrm{Hu}$, S.J., 1995. Introduction to Tibet Agriculture. 1st Edn., Sichuan Science and Technology Press, ISBN-10: 7-5364-2822-7, pp: 113.

Le, G.J., O. Delebarre, D. Beghin, E. Heumez an P. Pluchard, 1999. Nitrogen uptake and utilisation efficiency of two-row and six-row winter barley cultivars grown at two N levels. Eur. J. Agronomy, 10: 73-79. DOI: 10.1016/S1161-0301(98)00055-0

Li, J.Z., X.Q. Huang F. Heinrichs, M.W. Ganal and M.S. Röder, 2006. Analysis of QTLs for yield components, agronomic traits and disease resistance in an advanced backcross population of spring barley. Genome, 49: 454-466. DOI: 10.1139/G05-128 
Lu, J.Y., 1991. Molybdenum-antimony colorimetric determination of total phosphorus in soils. Environ. Sci. Technol., 4: 33-34.

Ma, D.Q., 2000. Barley Genetic Resources in Tibet. 1 st Edn., China Agriculture Press, ISBN-10: 7-109-06102-7.

Miralles, D.J. and R.A. Richards, 2000. Responses of leaf and tiller emergence and primordium initiation in wheat and barley to interchanged photoperiod. Annals Botany, 85: 655-663.

DOI: 10.1006/anbo.2000.1121

Papastylianou, I., 1995a. Yield components in relation to grain yield losses of barley fertilized with nitrogen. Eur. J. Agronomy, 4: 55-63. DOI: $10.1016 / \mathrm{S} 1161-0301(14) 80017-8$

Papastylianou, I., 1995b. The effects of seed rate and nitrogen fertilization on yield and yield components of two-row barley. Eur. J. Agronomy, 4: 237-243. DOI: 10.1016/S1161-0301(14)80050-6

Prado, S.A., J.M. Gallardo, R.A. Serrago, B.C. Kruk and D.J. Miralles, 2013. Comparative behavior of wheat and barley associated with field release and grain weight determination. Field Crops Res., 144: 28-33. DOI: $10.1016 /$ j.fcr.2012.12.018

Prystupa, P., R. Savin and G.A. Slafer, 2004. Grain number and its relationship with dry matter, $\mathrm{N}$ and $\mathrm{P}$ in the spikes at heading in response to $\mathrm{N} \times \mathrm{P}$ fertilization in barley. Field Crops Res., 90: 245-254. DOI: $10.1016 /$ j.fcr.2004.03.001
Reinhardt, D., G. Jansen, S. Seddig and B. EichlerLobermann, 2013. Temperature stress during flowering time affects yield and quality parameters of waxy barley. Landbauforschung Volkenrode, 63: 79-83. DOI: 10.3220/LBF 2013 79-84

Shuang, L. and J.D.U. Nisana, 2016. Determination of organic carbon in geochemical soil samples by potassium dichromate oxidation-external heating method. Anhui Chem. Industry, 42: 110-112.

Ugarte, C., D.F. Calderini and G.A. Slafer, 2007. Grain weight and grain number responsiveness to preanthesis temperature in wheat, barley and triticale. Field Crops Res., 100: 240-248. DOI: $10.1016 /$ j.fcr.2006.07.010

Wang, J., Z.M. Zhong and Z.H. Wang, 2014. Soil C/N distribution characteristics of alpine steppe ecosystem in Qinhai-Tibetan Plateau. Acta Ecol. Sinica, 34: 6679-6691. DOI: 10.5846 / stxb201302130263

Zou, W., T.M. Liu, D.Y. Kong, L. Tang and W.X. Cao, 2009. Simulation model on barley yield formation. Chinese J. Applied Ecol., 20: 396-402. PMID: 19459382 